\title{
Late Administration of Trastuzumab Emtansine Might Lead to Loss of Chance for Better Outcome in Patients with HER2-Positive Metastatic Breast Cancer
}

\author{
Luis Manso $^{a} \quad$ Alfonso Sanchez-Muñoz ${ }^{b}$ Isabel Calvo ${ }^{c}$ Yann Izarzugaza $^{d}$ Jessica Plata $^{e}$ \\ Cesar Rodriguez \\ a Medical Oncology Department, 12 de Octubre University Hospital, Madrid, Spain; \\ ${ }^{b}$ Clinical and Translational Cancer Research, Biomedical Research Institute of Málaga (IBIMA), Regional and Virgen de la Victoria \\ University Hospital, Málaga, Spain; \\ ${ }^{\mathrm{c}}$ Medical Oncology Department, HM Sanchinarro University Hospital, Madrid, Spain; \\ d Medical Oncology Department, Fundación Jiménez Díaz University Hospital, Madrid, Spain; \\ e Medical Oncology Department, Jaen University Hospital Complex, Jaén, Spain; \\ ${ }^{f}$ Medical Oncology Department, Salamanca Clinic University Hospital, Salamanca, Spain
}

\section{Keywords}

Antineoplastic agents · Oncologists .

Metastatic breast cancer . Trastuzumab emtansine . HER2-positive $\cdot$ Loss of chance

\section{Summary}

The optimal sequence of anti-human epidermal growth factor receptor 2 (HER2) therapies in metastatic breast cancer $(\mathrm{MBC})$ is still undetermined. Physicians must therefore make decisions based on clinical trials and their own experience for the best treatment sequence in these patients. The objective of this review is to summarize the efficacy and safety data for trastuzumab emtansine (T-DM1) in patients with MBC. Additionally, the concept of 'loss of chance for a better outcome' is investigated. It applies to patients who are not receiving the best possible treatment for their disease. Physicians should strive to offer the best possible care, although getting optimal results in each individual patient is not guaranteed. Lastly, the number of patients with MBC lost per treatment line is evaluated. We conclude that both concepts reinforce the importance of giving the most active treatments as soon as possible in the course of disease to secure the longest possible survival for HER2positive MBC patients.

(c) 2018 S. Karger GmbH, Freiburg

\section{Introduction}

Breast cancer is the most common cancer among European women, with an estimated incidence of 494,076 new cases ( $28 \%$ of all cancers) and 142,979 deaths (17\% of all cancer deaths) in 2012 [1]. In the USA, approximately 252,710 new cases of invasive breast cancer and 40,610 breast cancer deaths were expected to occur in 2017 [2]. However, breast cancer is a heterogeneous disease with different molecular subtypes defined by the biomarkers expressed.

Human epidermal growth factor receptor 2(HER2)-positive breast cancer accounts for approximately $15-20 \%$ of all invasive breast cancers $[3,4]$. While HER2-positive breast cancer was initially associated with shortened survival and an increased risk of disease recurrence and metastasis [5], the approval of anti-HER2targeted therapies in the last 20 years has changed the outlook for these patients [6-11]. Currently, trastuzumab, pertuzumab, lapatinib, and trastuzumab emtansine (T-DM1) are approved for use in different clinical settings in patients with HER2-positive breast cancer. All of the above inhibit the HER2 pathway, albeit using different strategies $[12,13]$. Trastuzumab is a recombinant humanized monoclonal antibody that binds the extracellular subdomain IV with internalization and degradation of the HER2 receptor. Pertuzumab is also a humanized monoclonal antibody that binds the HER2 extracellular subdomain II with the aim to prevent heterodimerization of HER2 with other members of the HER family. Lapatinib is a small molecule that inhibits intracellular HER1/HER2 tyrosine kinase to block the activation of their signaling pathways. Finally, T-DM1 is an antibody conjugate of trastuzumab with a

\section{KARGER}

() 2018 S. Karger GmbH, Freiburg

Fax +497614520714 
potent cytotoxic antimicrotubule agent (i.e., emtansine or DM1), which inhibits HER2 signaling and optimizes the delivery of chemotherapy at the tumor site.

Based on the efficacy and safety data of anti-HER2-targeted therapies [6-11], treatment guidelines for breast cancer issued by the American Society of Clinical Oncology (ASCO), the European Society for Medical Oncology (ESMO), and the National Comprehensive Cancer Network (NCCN) recommend administering antiHER2 therapy along with systemic chemotherapy or hormone therapy to patients with HER2-positive early, advanced, or metastatic disease [14-18]. However, the optimal sequence of all available anti-HER2 therapies is still undetermined [15]. Therefore, medical oncologists must base their decisions regarding the optimal treatment sequence on efficacy and safety data for anti-HER2 therapies from randomized clinical trials to reach the best clinical outcome while minimizing toxicity for each patient [19].

Our intention in this review is to summarize the current evidence for the efficacy and safety of T-DM1 in patients with metastatic breast cancer (MBC). Additionally, we investigate the concept of 'loss of chance for a better outcome', i.e., when a patient may not be receiving the best possible treatment for his/her disease. This concept, along with the continuous loss of $\mathrm{MBC}$ patients per treatment line, allows us to understand to what extent both issues may impact patient outcome.

\section{Efficacy of T-DM1}

The efficacy of T-DM1 has been well documented through an extensive research program. The most important trials are summarized below.

\section{Phase I Trials}

The first-in-human study with T-DM1 was reported in 2010 by Krop et al. [20]. T-DM1 was given to 24 patients with HER2-positive $\mathrm{MBC}$ who had received a median of 4 prior chemotherapy treatments. Dosing was started at $0.3 \mathrm{mg} / \mathrm{kg}$ on an every-3-weeks (q3w) cycle and escalated to $4.8 \mathrm{mg} / \mathrm{kg}$. Transient thrombocytopenia was the dose-limiting toxicity at $4.8 \mathrm{mg} / \mathrm{kg}$, and $3.6 \mathrm{mg} / \mathrm{kg}$ was identified as the maximum tolerated dose (MTD). The objective response rate (ORR) was $21 \%$ (5/24 patients; 4 of them at a dose level of $3.6 \mathrm{mg} / \mathrm{kg}$ ). The median duration of response was 10.5 months. 7 patients had stable disease for more than 6 months. A weekly dosing cohort was also evaluated starting at one-third of the $3.6 \mathrm{mg} / \mathrm{kg} \mathrm{q} 3 \mathrm{w}$ dosing schedule [21]. The MTD was determined to be $2.4 \mathrm{mg} / \mathrm{kg}$ weekly after 2 out of 3 patients at $2.9 \mathrm{mg} / \mathrm{kg}$ experienced grade 3 thrombocytopenia and grade 3 elevation of aspartate aminotransferase (AST). Of 28 patients treated with the weekly regimen, the ORR were reported in 13 (46\%) and the clinical benefit rate at 6 months was $57 \%$. However, grade 3 or worse adverse events (AEs) were more frequently observed with weekly dosing (68\%) than with the $\mathrm{q} 3 \mathrm{w}$ schedule (50\%), although patient numbers were small.

\section{Phase II Trials}

A total of 3 phase II trials have evaluated T-DM1 q3w as a single agent. A single-arm phase II pilot study of T-DM1 (TDM4258g) enrolled 112 patients whose disease had progressed on prior trastuzumab-based therapy. Patients had received a median of 5 prior treatment lines in the metastatic setting [22]. A confirmatory phase II single-arm study (TDM4374g) evaluated T-DM1 in a more heavily pretreated patient population [23]. In this study, 110 patients who had previously received anthracycline, capecitabine, taxane, lapatinib, and trastuzumab were enrolled. Both studies demonstrated promising activity for T-DM1, with an ORR of 26 and $35 \%$, respectively $[22,23]$. Median progression-free survival (PFS) was 4.6 and 6.9 months, respectively. Importantly, T-DM1 was shown to be well tolerated at the $\mathrm{q} 3 \mathrm{w}$ dosing.

Finally, a randomized phase II trial compared 137 patients with HER2-positive MBC, who had received T-DM1 or trastuzumab plus docetaxel (TD) as first-line treatment [24]. Baseline characteristics were similar between both treatment arms, although slightly more patients in the TD arm had prior treatment with trastuzumab ( 27 vs. $18 \%$ ) or a taxane ( 40 vs. $33 \%$ ) in the early disease setting. T-DM1 resulted in a significant prolongation of median PFS as compared with the control arm (14.2 vs. 9.2 months; $p=0.035)$, whereas there were no significant differences in terms of ORR ( $64 \%$ for T-DM1 vs. $58 \%$ in the control arm) or clinical benefit rate ( $75 \%$ for T-DM1 vs. $81 \%$ in the control arm). The toxicity profile of T-DM1 compared favorably with TD.

It is important to note that all 3 phase II trials retrospectively performed central analyses to confirm the HER2 status of their study populations. Hurvitz et al. [24] reported similar results when patients were confirmed to be HER2-positive. However, the studies by Krop et al. [23] and Burris et al. [22] had some discrepancies in that some patients who were locally identified as HER2-positive were found to be HER2-negative on central assessment, leading to better outcomes in the cohort of HER2-positive patients centrally confirmed.

\section{Phase III Trials}

The EMILIA trial was the first phase III trial to demonstrate the clinical efficacy of T-DM1 in the metastatic setting (table 1) [11]. This randomized, international, open-label clinical trial compared the efficacy of T-DM1 at a dose of $3.6 \mathrm{mg} / \mathrm{kg}$ iv every 21 days with oral lapatinib (1,250 $\mathrm{mg}$ daily) plus oral capecitabine $\left(2,000 \mathrm{mg} / \mathrm{m}^{2}\right.$ days 1-14 of a 21-day treatment cycle) in 991 patients with HER2positive, unresectable, locally advanced breast cancer (LABC) or $\mathrm{MBC}$, who had previously been treated with a taxane and trastuzumab. Both study arms were well balanced, with a high proportion of visceral metastasis ( $67 \%$ in the T-DM1 arm and $68 \%$ in the control arm).

Originally, the primary endpoint of the study was PFS assessed by an independent review panel, but later with data still masked, overall survival (OS) was included as a co-primary efficacy endpoint [25]. With a median follow-up of 19 months, treatment with T-DM1 improved PFS from 6.4 months to 9.6 months in the control arm with an absolute gain of 3.2 months (hazard ratio (HR) 
0.65; 95\% confidence interval (CI) 0.55-0.77; $\mathrm{p}<0.001$ ). Most importantly, patients in the T-DM1 arm experienced prolonged OS of 4.0 months (29.9 vs. 25.9 months; HR 0.75; p < 0.001) [25]. Moreover, the ORR was also improved with T-DM1 (44 vs. $31 \%$; p $<0.001$ ), mainly due to the greater number of partial responses. Median duration of response was also increased with T-DM1 compared with lapatinib plus capecitabine (12.6 vs. 6.5 months).

Of note, the benefit of T-DM1 was observed irrespective of the number of previous treatment lines given and even in patients whose disease progressed in less than 6 months after completing (neo-)adjuvant trastuzumab treatment. In addition, in the patientreported outcomes from the EMILIA trial, median time to symptom worsening was also significantly delayed in the T-DM1 arm compared with the lapatinib plus capecitabine arm (7.1 vs. 4.6 months; HR 0.796; $\mathrm{p}=0.012$ ) [26]. Interestingly, although patients with PIK3CA mutations had worse outcomes than those without mutations when treated with lapatinib and capecitabine (PFS 4.3 vs. 6.4 months), T-DM1-treated patients with PIK3CA mutations had a similar outcome than those without (PFS 10.9 vs. 9.8 months) [27]. These results suggest that the unique mechanism of action of T-DM1 may overcome PIK3CA mutation resistance. However, these observations need to be confirmed in additional trials.

The TH3RESA phase III trial compared T-DM1 versus treatment of physician's choice in LABC or MBC patients who had previously received both trastuzumab- and lapatinib-containing schedules in the metastatic setting. In this phase III, randomized, multicenter, open-label trial, patients needed to also have received a taxane in any setting and have documented investigator-assessed progression after being treated with 2 or more HER2-directed regimens in the advanced setting [28].

In the primary analysis, PFS was significantly improved with T-DM1 compared with the physician's choice (median: 6.2 vs. 3.3 months; HR 0.528; $\mathrm{p}<0.0001$ ). Results were first presented at the San Antonio Breast Cancer Symposium (SABCS) by Wildiers et al. [29] demonstrating a median OS improvement of 6.9 months (from 15.8 months in the control arm to 22.7 months in the T-DM1 arm; HR 0.68; $\mathrm{p}=0.0007$ ) and were later fully reported [30]. These results crossed the pre-specified OS efficacy-stopping boundary ( $\mathrm{HR}<0.75$ or $\mathrm{p}<0.012$ ). Of note, survival benefit was achieved in spite of 93 (47\%) of 198 patients in the physician's choice group having crossed over to T-DM1.

T-DM1 has also been tested as first-line treatment in the MARIANNE study [31]. This trial randomly assigned 1,095 patients with progressed or recurrent LABC or previously untreated HER2-positive $\mathrm{MBC}$ to receive T-DM1 plus pertuzumab ( $\mathrm{n}=363$ patients), T-DM1 plus placebo ( $\mathrm{n}=367$ patients), or trastuzumab plus taxane (docetaxel or paclitaxel; $\mathrm{n}=365$ patients).

After a median follow-up of 35 months, both T-DM1-containing regimens showed non-inferior PFS, but non-superiority, over trastuzumab plus taxane. The median PFS was 15.2 months in the T-DM1 plus pertuzumab arm (HR 0.87; 95\% CI 0.69-1.08; $\mathrm{p}=$ 0.14 ) and 14.1 months with T-DM1 alone (HR 0.91; 95\% CI 0.73$1.13 ; \mathrm{p}=0.31)$ compared with 13.7 months observed in the control arm. The OS data were not yet reached. The ORR was 64,60 , and $68 \%$ among the T-DM1 plus pertuzumab, T-DM1 alone, and control arms, respectively. However, the median duration of response was 21.2 months (95\% CI 15.8-29.3) in the T-DM1 plus pertuzumab arm, 20.7 months (95\% CI 14.8-25.0) in the T-DM1 alone arm, and 12.5 months (95\% CI 10.5-16.6) in the control arm. The trial also showed a benefit with T-DM1 over the control schedule with regard to toxicity and health-related quality of life outcomes; however, in spite of these results, T-DM1 is not indicated for firstline use.

In summary, results of the phase III trials EMILIA and TH3RESA suggest that T-DM1 should be the standard of choice in second or later lines in patients with HER2-positive MBC [32]. Based on this, in February 2013 and November 2013, the Food and Drug Administration (FDA) and the European Medicines Agency (EMA), respectively, approved T-DM1 as a single agent for the treatment of patients with HER2-positive LABC or MBC who had previously received trastuzumab and a taxane, separately or in combination. Additionally, patients should have received either prior therapy for locally advanced or metastatic disease or developed disease recurrence during or within 6 months of completing adjuvant therapy.

\section{Safety of Trastuzumab Emtansine}

Previous phase II studies have shown a favorable safety profile for T-DM1. Most of these studies were undertaken beyond firstline treatment; hence, most patients had prior exposure to trastuzumab. In spite of this, the risk of developing cardiotoxicity with T-DM1 was low and this was confirmed in subsequent phase III trials (table 2).

In the EMILIA trial [11], T-DM1 was better tolerated than the combination of lapatinib with capecitabine. Thus, patients treated with T-DM1 showed lower percentages of any-grade AEs compared with the control arm (96 vs. 98\%), grade 3 or higher AEs (41 vs. $57 \%$ ), serious AEs (16 vs. $18 \%$ ), and AEs with subsequent treatment discontinuation or dosage reduction (6 vs. $8 \% ; 15$ vs. $19 \%$, respectively). The proportion of patients with a left ventricular ejection fraction (LVEF) of less than $50 \%$ and at least $15 \%$ points below the baseline value was similar between both study arms (2\%).

The most frequent any-grade AEs reported with T-DM1 ( $\geq 25 \%$ of patients) were nausea (39\%), fatigue (35\%), and thrombocytopenia (28\%). The overall incidence of bleeding events was higher with T-DM1 than with lapatinib plus capecitabine (30 vs. 16\%), although rates of grade 3 or 4 bleeding events were around 1\% in both study arms. The most frequent grade 3 or 4 AEs observed with T-DM1 $(\geq 3 \%)$ were thrombocytopenia (13\%) and elevated serum concentrations of AST (4\%).

The TH3RESA trial included a more heavily pretreated population and showed similar results [28]. Any-grade AEs were reported in $94 \%$ of patients receiving T-DM1 and $89 \%$ of patients receiving physician's choice; however, a lower proportion of patients in the 
T-DM1 arm reported having grade 3 or higher AEs compared with the control arm ( 32 vs. $43 \%$ ), serious AEs (18 vs. $21 \%$ ), and AEs with subsequent treatment discontinuation or dosage reduction (7 vs. $11 \%$; 9 vs. $20 \%$, respectively). In the T-DM1 arm, the most frequent any-grade $\mathrm{AE}$ ( $\geq 25 \%$ of patients) was fatigue (27\%), whereas thrombocytopenia (5\%) and anemia (3\%) were the most frequent grade 3 or higher AEs observed. The proportion of patients with LVEF $<50 \%$ and a $\geq 15 \%$ decrease from baseline was $1 \%$ in both study arms. 3 treatment-related deaths occurred in the T-DM1 arm due to hepatic encephalopathy, pneumonitis, and subarachnoid hemorrhage. The grade 5 subarachnoid hemorrhage was associated with grade 4 thrombocytopenia; however, this patient was also concomitantly receiving anticoagulant therapy. Overall, $2 \%$ of patients in the T-DM1 arm had grade 3 or worse hemorrhage compared with $<1 \%$ in the control arm.

Although in the MARIANNE trial T-DM1 either alone or in combination with pertuzumab did not show superior efficacy over trastuzumab plus taxane, it is worth mentioning the safety profile observed in these patients due to the high number of participants [31]. Any-grade AEs occurred in 99\% of patients in the 3 arms. However, a lower proportion of patients in both T-DM1 arms were reported as having grade 3 or higher AEs compared with the control arm (45\% with T-DM1, 46\% with T-DM1 plus pertuzumab, and $54 \%$ with trastuzumab plus taxane). The rate of LVEF $<50 \%$ with a $\geq 15 \%$ decrease from baseline was $1 \%$ with T-DM1, $2.5 \%$ with T-DM1 plus pertuzumab, and $4.5 \%$ with trastuzumab plus taxane. Deaths due to an $\mathrm{AE}$ were similar across treatment arms $(1-2 \%)$.

The most commonly reported any-grade AEs with T-DM1 alone ( $\geq 25 \%$ of patients) were nausea $(47 \%)$, headache $(32 \%)$, epistaxis $(31 \%)$, pyrexia $(28 \%)$, and diarrhea $(25 \%)$. The addition of pertuzumab to T-DM1 did not substantially increase the incidence of these toxicities, except for diarrhea (from 25\% to 48\%) and vomiting (from $22 \%$ to $30 \%$ ). Grade 3 or higher AEs observed in $\geq 3 \%$ in both T-DM1 arms were AST and alanine transaminase increase, thrombocytopenia, anemia, hypertension, and neutropenia. Only diarrhea increased substantially with the addition of pertuzumab (from $<1 \%$ to $3 \%$ ).

In summary, the most frequently reported high-grade AEs associated with the administration of T-DM1 were laboratory abnormalities such as thrombocytopenia, transaminase elevation, and anemia. It is important to note that the mechanism by which thrombocytopenia occurs is unknown. It usually appears during the first 2 cycles of T-DM1 and is mostly managed with dose reductions. Transaminase elevations are usually transient and without any clinical impact. Lastly, although cardiotoxicity remains a clinically significant toxicity for all anti-HER2 therapies, novel agents such as T-DM1 seem to have a reduced potential to cause it [33]. Nevertheless, these findings should be considered with caution as patients from clinical trials are highly selected and not always fully representative of patients seen in clinical practice. As a result, LVEF monitoring should be performed periodically during treatment with T-DM1.

\section{Loss of Chance for a Better Outcome}

\section{Concept}

During the last decade, the concept of 'loss of chance for a better outcome' has emerged. Based on it, the most common conditions that may suggest a loss of chance for a better outcome include lack of information, lack of or delay in diagnosis, delay in action, and fault in medical assessment [34].

Medicine is always surrounded by causal uncertainty. The same treatment may act differently depending on the patient who is receiving it. Therefore, physicians are obliged to give the best possible treatment even though they cannot ensure that this treatment will achieve an optimal result in any individual patient. The verdict of loss of chance depends on showing to what extent a patient's outcome would have been improved by earlier diagnosis or if they had been better treated.

\section{Loss of Cancer Patients per Treatment Line}

In $\mathrm{MBC}$, there is a continuous decline in the number of patients per treatment line, and medical oncologists are often unaware of the importance of this decline. In a retrospective observational study, Hao et al. [35] analyzed the treatment patterns and clinical outcomes among 18,059 US women with MBC between 2008 and 2011. Of the eligible population, 15\% were treated with anti-HER 2 agents, $71 \%$ were treated with other anticancer drugs, and $14 \%$ remained untreated. Patients treated with anti-HER2 agents were, on average, the youngest cohort (55.6 vs. 60.1 vs. 62.3 years, respectively; $\mathrm{p}<0.001$ ). Interestingly, over $80 \%$ of patients in the HER2-targeted agent cohort received a first-line therapy (including a HER2-targeted agent in 95\%) during follow-up, 15\% received a second line (including a HER2-targeted agent in 73\%), and only $3 \%$ received a third line (including a HER2-targeted agent in 51\%).

In another retrospective observational study performed between 2005 and 2009 [36], 7,767 patients with MBC were identified and received a first-line treatment with chemotherapy or biologic therapies. The mean patient age was 58 years; $36 \%$ had received prior radiation therapy, and $23 \%$ had undergone breast surgery. Overall, only $52 \%$ received a second-line therapy, $26 \%$ received a third-line therapy, and $14 \%$ received a fourth-line therapy. Moreover, within each group of patients with a similar length of followup, those with a greater number of lines had greater cumulative costs. This suggests that patients who progressed in earlier lines of therapy had higher costs during the same time than those who did not progress as quickly.

The Spanish observational study CASCADE identified 443 patients with MBC in 13 public hospitals between 2007 and 2008 and followed them up to the end of 2013 (5-7 years of follow-up) or death $[37,38]$. Tumor classification according to receptor immunotypes included HER2-negative hormone receptor(HR)-negative (16\%), HER2-negative HR-positive (44\%), HER2-positive HR-negative (12\%), and HER2-positive HR-positive (17\%), while immunophenotype was unknown in $11 \%$ of patients. The study observed a continuous decline in patient numbers from line to line; treat- 
Table 1. Summary of efficacy outcomes of T-DM1 in main phase III clinical trials

\begin{tabular}{|c|c|c|c|c|c|c|c|}
\hline Study [ref.] & Study population & Treatment arms & Patients, $\mathrm{n}$ & $\begin{array}{l}\text { Median FU, } \\
\text { months }\end{array}$ & $\begin{array}{l}\text { Median PFS, } \\
\text { months; p }\end{array}$ & $\begin{array}{l}\text { Median OS, } \\
\text { months; } \mathrm{p}\end{array}$ & $\begin{array}{l}\text { ORR, \%; } \\
\text { p }\end{array}$ \\
\hline $\begin{array}{l}\text { EMILIA } \\
\qquad[11,25]\end{array}$ & $\begin{array}{l}\text { ABC HER2+ with } \\
\text { previous trastuzumab } \\
\text { and taxane }\end{array}$ & $\begin{array}{l}\text { T-DM1 vs. lapatinib plus } \\
\text { capecitabine }\end{array}$ & $495 / 496$ & 19 & $\begin{array}{l}9.6 \text { vs. } 6.4 \\
p<0.001\end{array}$ & $\begin{array}{l}29.9 \text { vs. } 25.9 \\
p<0.001\end{array}$ & $\begin{array}{l}44 \text { vs. } 31 \\
\text { p }<0.001\end{array}$ \\
\hline $\begin{array}{r}\text { TH3RESA } \\
{[28,29]}\end{array}$ & $\begin{array}{l}\text { ABC HER2+ with } \\
\text { previous trastuzumab, } \\
\text { lapatinib, and taxane }\end{array}$ & $\begin{array}{l}\text { T-DM1 vs. physician's } \\
\text { choice }\end{array}$ & $404 / 198$ & 7 & $\begin{array}{l}6.2 \text { vs. } 3.3 \\
p<0.0001\end{array}$ & $\begin{array}{l}22.7 \text { vs. } 15.8 \\
\mathrm{p}<0.001\end{array}$ & $\begin{array}{l}31 \text { vs. } 9 \\
p<0.0001\end{array}$ \\
\hline $\begin{array}{l}\text { MARIANNE } \\
{[31]}\end{array}$ & $\begin{array}{l}\text { ABC HER2+ without } \\
\text { previous treatment at } \\
\text { first-line }\end{array}$ & $\begin{array}{l}\text { T-DM1 plus placebo vs. } \\
\text { T-DM1 plus pertuzumab } \\
\text { vs. trastuzumab plus taxane }\end{array}$ & $367 / 363 / 365$ & 35 & $\begin{array}{l}14.1 \text { vs. } 15.2 \\
\text { vs. } 13.7 \\
\mathrm{p}=\mathrm{ns}\end{array}$ & NR/NR/NR & $\begin{array}{l}60 \text { vs. } 64 \\
\text { vs. } 68\end{array}$ \\
\hline
\end{tabular}

$\mathrm{ABC}=$ Advanced breast cancer; $\mathrm{FU}=$ follow-up; HER2 $+=$ human epidermal growth factor receptor 2-positive; NR = not reported; ns = not specified;

$\mathrm{ORR}=$ overall response rate; $\mathrm{OS}=$ overall survival; $\mathrm{PFS}=$ progression-free survival; $\mathrm{T}-\mathrm{DM} 1$ = trastuzumab emtansine.

Table 2. Safety data for T-DM1 in main phase III clinical trials

\begin{tabular}{|c|c|c|c|c|c|c|c|}
\hline \multirow[t]{2}{*}{ Grade $\geq 3^{\mathrm{a}}$} & \multicolumn{2}{|c|}{ EMILIA [11], n (\%) } & \multicolumn{2}{|c|}{ TH3RESA $[28,29], \mathrm{n}(\%)$} & \multicolumn{3}{|c|}{ MARIANNE [31], n (\%) } \\
\hline & $\begin{array}{l}\text { L plus C } \\
(\mathrm{n}=488)\end{array}$ & $\begin{array}{l}\text { T-DM1 } \\
(\mathrm{n}=490)\end{array}$ & $\begin{array}{l}\text { Physician's } \\
\text { Choice } \\
(\mathrm{n}=184)\end{array}$ & $\begin{array}{l}\text { T-DM1 } \\
(\mathrm{n}=403)\end{array}$ & $\begin{array}{l}\mathrm{H} \text { plus T } \\
(\mathrm{n}=353)\end{array}$ & $\begin{array}{l}\text { T-DM1 } \\
(\mathrm{n}=361)\end{array}$ & $\begin{array}{l}\text { T-DM1 plus } \mathrm{P} \\
(\mathrm{n}=366)\end{array}$ \\
\hline \multicolumn{8}{|l|}{ Non-hematologic } \\
\hline Abdominal pain & - & - & $5(3)$ & $5(1)$ & - & - & - \\
\hline Diarrhea & $101(21)$ & $8(2)$ & $8(4)$ & $3(<1)$ & $15(4)$ & $1(<1)$ & $9(3)$ \\
\hline Fatigue & $17(4)$ & $12(2)$ & - & - & - & - & - \\
\hline Hand-foot syndrome & $80(16)$ & $0(0)$ & - & - & - & - & - \\
\hline Hypertension & - & - & - & - & $11(3)$ & $16(4)$ & $18(5)$ \\
\hline Vomiting & $22(5)$ & $4(1)$ & - & - & - & - & - \\
\hline \multicolumn{8}{|l|}{ Hematologic } \\
\hline Anemia & - & - & $5(3)$ & $11(3)$ & $10(3)$ & $17(5)$ & $22(6)$ \\
\hline ALT increase & - & - & - & - & $3(1)$ & $16(4)$ & $19(5)$ \\
\hline AST increase & $4(1)$ & $21(4)$ & - & - & $1(<1)$ & $24(7)$ & $11(3)$ \\
\hline Febrile neutropenia & - & - & $7(4)$ & $1(<1)$ & $23(7)$ & $0(0)$ & $0(0)$ \\
\hline Hypokalemia & $20(4)$ & $11(2)$ & - & - & - & - & - \\
\hline Leukopenia & - & - & $5(3)$ & $1(<1)$ & - & - & - \\
\hline Neutropenia & $21(4)$ & $10(2)$ & $29(16)$ & $10(2)$ & $70(20)$ & $16(4)$ & $10(3)$ \\
\hline Thrombocytopenia & $1(<1)$ & $63(13)$ & $3(2)$ & $19(5)$ & $0(0)$ & $23(6)$ & $29(8)$ \\
\hline
\end{tabular}

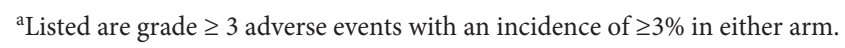

$\mathrm{ALT}=$ Alanine aminotransferase; $\mathrm{AST}=$ aspartate aminotransferase; $\mathrm{C}=$ capecitabine; $\mathrm{H}=$ trastuzumab; $\mathrm{L}=$ lapatinib; $\mathrm{P}=$ pertuzumab; $\mathrm{T}=$ taxane; $\mathrm{T}-\mathrm{DM} 1$ = trastuzumab emtansine. ment varied according to tumor immunotype, with the worst outcome for patients with triple-negative tumors (32\% of HER2-negative HR-negative patients received a fourth line vs. $43 \%$ of HER2negative HR-positive patients, $42 \%$ of HER2-positive HR-negative patients, and 40\% of HER2-positive HR-positive patients). Also, it was observed that median PFS decreased by half from first-line to third-line treatment among all immunotypes. Interestingly, a statistically significant OS increase of 15 months was seen between first- and/or second-line responders and non-responders in the overall study population. Thus, the results of the CASCADE study support the administration of the most active treatment options as early as possible.

\section{Use of Anti-HER2-Targeted Therapies in the First Line}

RegistHER was a US population-based, prospective, observational study that evaluated 1,023 patients with HER2-positive breast cancer diagnosed 2003-2006 and followed them until 2009 or death. Overall, $87 \%$ of patients were treated with trastuzumab in the first-line setting. Median OS was longer in patients who were treated with first-line trastuzumab than in patients who were not (35.9 vs. 31.4 months) [39]. However, in the more recent SystHERs study that started enrollment in 2012 and already has data available for 872 patients with HER2-positive MBC, $96 \%$ of de novo patients and $93 \%$ of recurrent patients received an anti-HER2-based therapy as first-line treatment, which resulted in a significant improvement [40]. 
In the European EU-5 study, treatment pattern according to HR and HER2 status was evaluated in 152,311 newly diagnosed patients with MBC between 2008 and 2010 in France, Germany, Italy, Spain, and the UK via a physician survey [41]. It was found that the use of HER2-targeted therapy greatly varied between countries. While anti-HER2 agents were most frequently used in France, they were the least frequently used in the UK. Focusing on HER2-positive HR-positive MBC patients, HER2-based therapy in the firstline setting was received by $27 \%$ of patients in the UK, $36 \%$ in Spain, $37 \%$ in Italy, $41 \%$ in Germany, and $64 \%$ in France. These percentages increased notably in HER-positive HR-negative MBC patients, being $51 \%$ in UK, 58\% in Germany, 66\% in Italy, $68 \%$ in Spain, and $75 \%$ in France. In summary, on average, around half of the patients with HER2-positive HR-positive MBC and one-third of patients with HER2-positive HR-negative MBC patients did not receive anti-HER2 therapy in the first line. Almost 10 years later, it could be expected that this situation would have improved.

\section{Use of T-DM1 in the Real-World Setting}

To date, there are very few reported studies describing the use of T-DM1 in the real-world setting. Dzimitrowicz et al. [42] assessed the efficacy of T-DM1 in routine clinical practice in a patient population identified via electronic pharmacy records. Overall, $96 \%$ of patients received both prior trastuzumab and pertuzumab, and $23 \%$ also received lapatinib before T-DM1. Of 78 patients available for analysis, $32 \%$ received T-DM1 as first or second line, $21 \%$ as third line, and $48 \%$ as forth line or beyond. Median duration on therapy was 4 months, and response rate was $18 \%$. These results are less favorable than those reported in the EMILIA trial [11], although more comparable with results obtained in TH3RESA and the TDM4258g and TDM4374g trials which enrolled more heavily pretreated patients [22, 23, 28].

The T-DM1 Patient Access Study (T-PAS) reported data for the first 215 enrolled patients. T-DM1 was given after a median number of 8 prior systemic therapies for MBC (range: 3-23). Median T-DM1 treatment duration was 5 months, and ORR was $26 \%$. Overall, $19 \%$ of patients received more than 18 cycles. It was concluded that T-DM1 was efficacious in the real-world setting with no safety concerns.

\section{Conclusion}

All patients with HER2-positive MBC who relapse after adjuvant anti-HER2 therapy should be considered for further antiHER2 therapy. The combination of chemotherapy, trastuzumab, and pertuzumab is superior to the combination of chemotherapy plus trastuzumab in patients with previously untreated HER2-positive breast cancer. Hence, this schedule is approved to be given to patients with early-stage breast cancer at high risk of recurrence or metastatic disease. However, the dual blockade with both trastuzumab and pertuzumab associated with chemotherapy is not currently supported beyond the first line once this schedule has already been given. Additionally, for selected patients with HER2positive and HR-positive breast cancer, endocrine treatment with either trastuzumab or lapatinib may be an acceptable first-line treatment.

After a first-line trastuzumab-based therapy, T-DM1 has been demonstrated in 2 randomized phase III trials to be superior to lapatinib plus capecitabine as well as to the treatment of physician's choice in terms of PFS and OS in HER2-positive MBC patients. Moreover, in both trials, T-DM1 was better tolerated than the control treatments. The most frequent high-grade AEs associated with T-DM1 were laboratory abnormalities easily managed with dose modifications and without any clinical impact.

In recent years, physicians have become increasingly aware of the high number of patients with MBC that are lost per treatment line. In the Spanish observational study CASCADE, it was observed that only $40 \%$ of patients diagnosed with $\mathrm{MBC}$ will receive a fourth line of treatment. In addition, it was observed that median PFS decreased by about $50 \%$ from first-line to third-line treatment. It is reasonable to infer that both issues may impact the OS of these patients. Lastly, the newly developed concept of 'loss of chance for a better outcome' reinforces the importance of knowing that physicians must not delay the use of the most effective treatments but deploy them as soon as indicated.

\section{Acknowledgment}

The authors acknowledge the assistance of Dr. Beatriz Gil-Alberdi from HealthCo (Madrid, Spain) for her help in preparing the first draft of this manuscript. The necessary scientific meetings along with medical writing services were supported financially by Roche Farma Spain. All authors approved the final version of the submitted manuscript.

\section{Ethical Statement}

The study has been performed in accordance with the ethical standards of the Declaration of Helsinki and its later amendments. This article does not contain any studies with human participants or animals performed by any of the authors.

\section{Disclosure Statement}

The authors declare that they do not have any conflicts of interest that could inappropriately influence their work. 


\section{References}

1 Ferlay J, Soerjomataram I, Ervik M, et al: GLOBOCAN 2012 v1.0, Cancer Incidence and Mortality Worldwide IARC CancerBase No. 11. Lyon, International Agency for Research on Cancer, 2013. globocan.iarc.fr (accessed March 2017).

2 DeSantis CE, Ma J, Goding Sauer A, et al: Breast cancer statistics, 2017, racial disparity in mortality by state. CA Cancer J Clin 2017;67:439-448.

3 Owens MA, Horten BC, Da Silva MM: HER2 amplification ratios by fluorescence in situ hybridization and correlation with immunohistochemistry in a cohort of 6556 breast cancer tissues. Clin Breast Cancer 2004;5:63-69.

4 Varga Z, Noske A, Ramach C, et al: Assessment of HER2 status in breast cancer: overall positivity rate and accuracy by fluorescence in situ hybridization and immunohistochemistry in a single institution over 12 years: a quality control study. BMC Cancer 2013;13:615.

5 Slamon DJ, Clark GM, Wong SG, et al: Human breast cancer: correlation of relapse and survival with amplification of the HER-2/neu oncogene. Science 1987;235: 177-182.

6 Slamon D, Eiermann W, Robert N, et al.; Breast Cancer International Research Group: Adjuvant trastuzumab in HER2-positive breast cancer. N Engl J Med 2011;365:1273-1283.

7 Slamon DJ, Leyland-Jones B, Shak S, et al: Use of chemotherapy plus a monoclonal antibody against HER2 for metastatic breast cancer that overexpresses HER2. N Engl J Med 2001;344:783-792.

$\checkmark$ Dawood S, Broglio K, Buzdar AU, et al: Prognosis of women with metastatic breast cancer by HER2 status and trastuzumab treatment: an institutional-based review. J Clin Oncol 2010;28:92-98.

-9 Swain SM, Kim SB, Cortes J, et al: Pertuzumab, trastuzumab, and docetaxel for HER2-positive metastatic breast cancer (CLEOPATRA study): overall survival results from a randomised, double-blind, placebo-controlled, phase 3 study. Lancet Oncol 2013;14:461-471.

10 Geyer CE, Forster J, Lindquist D, et al: Lapatinib plus capecitabine for HER2-positive advanced breast cancer. N Engl J Med 2006;355:2733-2743.

11 Verma S, Miles D, Gianni L, et al.; EMILIA Study Group: Trastuzumab emtansine for HER2-positive advanced breast cancer. N Engl J Med 2012;367:17831791.

12 Jackisch C, Lammers P, Jacobs I: Evolving landscape of human epidermal growth factor receptor 2-positive breast cancer treatment and the future of biosimilars. Breast 2017;32:199-216.

13 Jiang H, Rugo HS: Human epidermal growth factor receptor 2 positive (HER2+) metastatic breast cancer: how the latest results are improving therapeutic options. Ther Adv Med Oncol 2015;7:321-339.

14 National Comprehensive Cancer Network Clinical Practice Guidelines in Oncology (NCCN Guidelines), Breast Cancer (version 2.2017). 2017. www.nccn.org/ professionals/physician_gls/pdf/breast.pdf (accessed October 2017).

15 Cardoso F, Costa A, Senkus E, et al: 3rd ESO-ESMO International Consensus Guidelines for Advanced Breast Cancer (ABC 3). Ann Oncol 2017;28:16-33.

16 Denduluri N, Somerfield MR, Eisen A, et al: Selection of optimal adjuvant chemotherapy regimens for human epidermal growth factor receptor 2 (HER2)negative and adjuvant targeted therapy for HER2positive breast cancers: an American Society of Clinical Oncology guideline adaptation of the Cancer Care Ontario Clinical Practice Guideline. J Clin Oncol 2016; 34:2416-2427.
7 Giordano SH, Temin S, Kirshner JJ, et al.; American Society of Clinical Oncology: Systemic therapy for patients with advanced human epidermal growth factor receptor 2-positive breast cancer: American Society of Clinical Oncology clinical practice guideline. J Clin Oncol 2014;32:2078-2099.

18 Senkus E, Kyriakides S, Ohno S, et al.; ESMO Guidelines Committee: Primary breast cancer: ESMO Clinical Practice Guidelines for diagnosis, treatment and follow-up. Ann Oncol 2015;26(suppl 5):v8-30.

19 Ricci F, Le Tourneau C: Trastuzumab emtansine in HER2-positive metastatic breast cancer: what is the best sequence? Chin Clin Oncol 2018;7:3.

20 Krop IE, Beeram M, Modi S, et al: Phase I study of trastuzumab-DM1, an HER2 antibody-drug conjugate, given every 3 weeks to patients with HER2-positive metastatic breast cancer. J Clin Oncol 2010;28:26982704.

21 Beeram M, Krop IE, Burris HA, et al: A phase 1 study of weekly dosing of trastuzumab emtansine (T-DM1) in patients with advanced human epidermal growth factor 2-positive breast cancer. Cancer 2012;118:57335740.

22 Burris HA 3rd, Rugo HS, Vukelja SJ, et al: Phase II study of the antibody drug conjugate trastuzumabDM1 for the treatment of human epidermal growth factor receptor 2 (HER2)-positive breast cancer after prior HER2-directed therapy. J Clin Oncol 2011;29: 398-405.

23 Krop IE, LoRusso P, Miller KD, et al: A phase II study of trastuzumab emtansine in patients with human epidermal growth factor receptor 2-positive metastatic breast cancer who were previously treated with trastuzumab, lapatinib, an anthracycline, a taxane, and capecitabine. J Clin Oncol 2012;30:3234-3241.

24 Hurvitz SA, Dirix L, Kocsis J, et al: Phase II randomized study of trastuzumab emtansine versus trastuzumab plus docetaxel in patients with human epidermal growth factor receptor 2-positive metastatic breast cancer. J Clin Oncol 2013;31:1157-1163.

25 Dieras V, Miles D, Verma S, et al: Trastuzumab emtansine versus capecitabine plus lapatinib in patients with previously treated HER2-positive advanced breast cancer (EMILIA): a descriptive analysis of final overall survival results from a randomised, open-label, phase 3 trial. Lancet Oncol 2017;18:732-742.

26 Welslau M, Dieras V, Sohn JH, et al: Patient-reported outcomes from EMILIA, a randomized phase 3 study of trastuzumab emtansine (T-DM1) versus capecitabine and lapatinib in human epidermal growth factor receptor 2-positive locally advanced or metastatic breast cancer. Cancer 2014;120:642-651.

27 Baselga J, Verma S, Ro J, et al: Relationship between tumor biomarkers (BM) and efficacy in EMILIA, a phase III study of trastuzumab emtansine (T-DM1) in HER2-positive metastatic breast cancer (MBC). Cancer Res 2013;73(suppl):abstr LB-63.

28 Krop IE, Kim SB, Gonzalez-Martin A, et al.; on behalf of the TH3RESA study collaborators: Trastuzumab emtansine versus treatment of physician's choice for pretreated HER2-positive advanced breast cancer (TH3RESA): a randomised, open-label, phase 3 trial. Lancet Oncol 2014;15:689-699.
Wildiers H, Kim SB, Gonzalez-Martin A, LoRusso PM Ferrero JM, Badovinac-Crnjevic T, Yu R, Smitt M, Krop IE: Trastuzumab emtansine (T-DM1) improves overall survival versus treatment of physician's choice in patients with previously treated HER2-positive metastatic breast cancer: final overall survival results from the phase 3 TH3RESA study. San Antonio Breast Cancer Symposium, San Antonio, TX, 2015, abstr S5-05.

30 Krop IE, Kim SB, Martin AG, et al: Trastuzumab emtansine versus treatment of physician's choice in patients with previously treated HER2-positive metastatic breast cancer (TH3RESA): final overall survival results from a randomised open-label phase 3 trial. Lancet Oncol 2017;18:743-754.

31 Perez EA, Barrios C, Eiermann W, et al: Trastuzumab emtansine with or without pertuzumab versus trastuzumab plus taxane for human epidermal growth factor receptor 2-positive, advanced breast cancer: primary results from the phase III MARIANNE study. J Clin Oncol 2017;35:141-148

32 Metzger-Filho O, Winer EP: Breast cancer: T-DM1 an important agent in the history of breast cancer management. Nat Rev Clin Oncol 2017;14:651-652.

33 Ponde NF, Lambertini M, de Azambuja E: Twenty years of anti-HER2 therapy-associated cardiotoxicity. ESMO Open 2016;1:e000073.

34 Nguyen TD: The concept of loss of chance: a major evolution in the definition of damage or how to prevent litigation for loss of chance? (Article in French). Cancer Radiother 2016;20:411-415.

35 Hao Y, Meyer N, Song X, et al: Treatment patterns and survival in metastatic breast cancer patients by tumor characteristics. Curr Med Res Opin 2015;31:275-288.

36 Ray S, Bonthapally V, McMorrow D, et al: Patterns of treatment, healthcare utilization and costs by lines of therapy in metastatic breast cancer in a large insured US population. J Comp Eff Res 2013;2:195-206.

37 Zamora P, Servitja S, Santaballa A, et al: CASCADE study: treatment and clinical outcomes of metastatic breast cancer by tumor immunophenotypes. Cancer Res 2016;76:abstr P3-07-39-P3-07-39.

38 Arias LDP, Teijido PG, Servitja S, et al: CASCADE study: pronounced decline in treatment efficacy through the metastatic life of breast cancer patients. Ann Oncol 2016;27:248P.

39 Rugo H, Kaufman P, Tan-Chiu E, et al: Survival of patients with HER2 + metastatic breast cancer and use of trastuzumab following progression: analysis of RegistHER. Cancer Res 2009;69:3142.

40 Cobleigh M, Yardley D, Brufsky A, et al: Baseline (BL) characteristics, treatment (tx) patterns, and outcomes in patients with hormone receptor $(\mathrm{HR})+\mathrm{vs} \mathrm{HR}-$ HER2+ disease from the SystHERs registry. Cancer Res 2017;77:abstr P50808.

41 DeKoven M, Bonthapally V, Jiao X, et al: Treatment pattern by hormone receptors and HER2 status in patients with metastatic breast cancer in the UK, Germany, France, Spain and Italy (EU-5): results from a physician survey. J Comp Eff Res 2012;1:453-463.

42 Dzimitrowicz H, Berger M, Vargo C, et al: T-DM1 activity in metastatic human epidermal growth factor receptor 2-positive breast cancers that received prior therapy with trastuzumab and pertuzumab. J Clin Oncol 2016;10:3511-3517. 